\title{
Montagem, calibração e testes de um sistema de medidas elétricas em função da temperatura e da pressão parcial de oxigênio
}

\section{(An experimental setup for electrical measurements as a function of temperature and partial pressure of oxygen)}

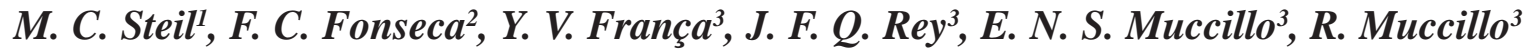 \\ ${ }^{1}$ Laboratoire de Cristallochimie et de Physicochimie du Solide \\ UMR CNRS 8012, ENSCL, Université de Science et Technologie de Lille \\ B.P. 108, 59652 Villeneuve d'Ascq Cedex, França. \\ ${ }^{2}$ Instituto de Física, Universidade de S. Paulo \\ C.P. 66318, S. Paulo, SP, Brasil, 05315-970. \\ ${ }^{3}$ CMDMC, CCTM-Instituto de Pesquisas Energéticas e Nucleares \\ C.P. 11049, Pinheiros, S. Paulo, SP, Brasil, 05422-970.
}

\begin{abstract}
Resumo
Neste trabalho são descritas a montagem e a calibração de um sistema de medidas elétricas com o controle da pressão parcial de oxigênio. Este sistema permite a realização de medidas elétricas desde a temperatura ambiente até $1000^{\circ} \mathrm{C}$ em ampla faixa de pressões parciais de oxigênio entre $10^{-6}$ e $1 \mathrm{~atm}$. O sistema utiliza as propriedades eletroquímicas dos eletrólitos sólidos à base de zircônia estabilizada para produzir e detectar pressões parciais de oxigênio com boa precisão. As calibrações e testes realizados, compreendendo a verificação das leis de Nernst e de Faraday, evidenciaram o bom funcionamento dos dispositivos construídos. A montagem apresentada neste trabalho, utilizada em conjunto com um sistema de medidas de espectroscopia de impedância, permite a realização de diversos estudos acerca das propriedades elétricas de materiais. Também são apresentados neste trabalho dois exemplos destes estudos, que consistem na investigação das propriedades elétricas de eletrólitos sólidos à base de zircônia e de varistores à base de óxido de estanho.
\end{abstract}

Palavras-chave: propriedades elétricas, espectroscopia de impedância, dependência com $\mathrm{p}_{\mathrm{O}_{2}}$, eletrólitos sólidos, varistores.

\begin{abstract}
The set-up and calibration of a system for electrical measurements under controlled oxygen partial pressure is described. The system allows for the electrical characterization of materials from room temperature to $1000{ }^{\circ} \mathrm{C}$ over a wide range of oxygen partial pressures ranging from $10^{-6}$ to 1 atm. The system takes advantage of the electrochemical properties of the zirconia based solid electrolytes to produce and detect controlled oxygen partial pressures with accuracy. The calibrations and tests, and also the experimental verifications of both Nernst and Faraday laws, assure that the whole experimental arrangement works properly. Such a set-up along with an impedance spectroscopy measurement system provides a useful tool to study the electrical properties of different materials. In this work, two examples concerning the electrical characterization of zirconia based electrolytes and tin oxide varistors are also presented.

Keywords: electrical properties, impedance spectroscopy, $p_{O_{2}}$ dependence, solid electrolytes, varistors.
\end{abstract}

\section{INTRODUÇÃOO}

A realização de medidas de condutividade elétrica em função da pressão parcial de oxigênio e da temperatura são importantes tanto do ponto de vista científico quanto tecnológico no estudo de materiais. Estas medidas permitem, por exemplo, estudar o regime de ionicidade de um condutor iônico, determinando-se a faixa de temperatura e pressão parcial de oxigênio na qual a condutividade iônica permanece constante. Portanto, este tipo de caracterização é importante para se definir as possíveis aplicações de materiais em dispositivos eletroquímicos. Do ponto de vista científico, estas medidas também permitem a identificação dos diferentes tipos de portadores de carga responsáveis pelo transporte elétrico em um material. As condutividades eletrônica e iônica variam de maneira diferente com a temperatura e a composição da atmosfera em equilíbrio com o material. Além de diferenciar eventuais contribuições parciais de diferentes tipos de portadores de carga em um material, estas medidas possibilitam também identificar a natureza dos portadores de carga, por exemplo, a determinação dos portadores em um semicondutor (tipo p ou n) [1]. Portanto, este tipo de caracterização elétrica 


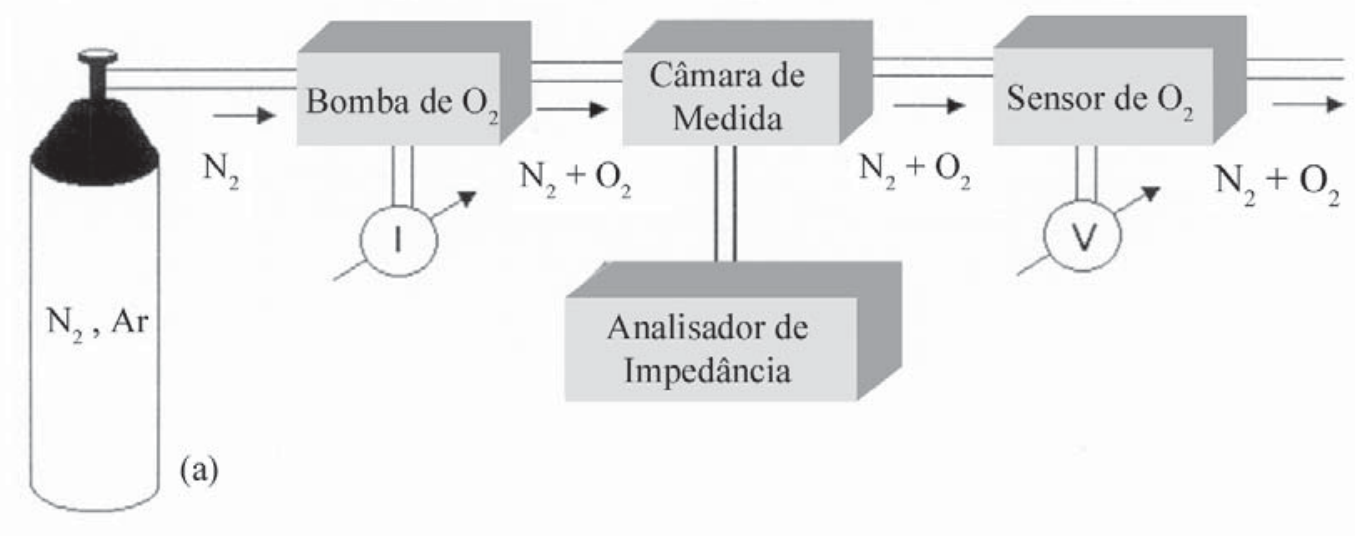

Figura 1: Diagrama esquemático do sistema de medidas: (a) cilindro do gás vetor, (b) bomba de oxigênio e fonte de corrente, (c) célula de medidas e o analisador de impedância, e (d) sensor de oxigênio e milivoltímetro.

[Figure 1: Schematics of the experimental setup:(a) gas cylinder, (b) oxygen pump and current supply, (c) measurement cell and impedance analyzer, and (d) oxygen sensor and millivoltmeter.]

pode ser muito útil no estudo de condutores mistos (materiais que apresentam condução elétrica eletrônica e iônica) e de novos condutores.

A condutividade elétrica total $(\sigma)$ corresponde à soma das condutividades parciais que são atribuídas a um determinado portador de carga. A fração da condutividade total devida ao transporte de um determinado portador de carga é denominada número de transporte $(\mathrm{t})$, e a soma de todos os números de transporte é igual a 1. Quando a maior parte da corrente elétrica é transportada por elétrons ou buracos (electron holes) o material é conhecido como condutor eletrônico $\left(\mathrm{t}_{\mathrm{e}} \approx 1\right)$; quando a densidade e a mobilidade dos defeitos iônicos é suficientemente alta para que a corrente iônica tenha valores muito superiores à eletrônica tem-se um condutor iônico $\left(\mathrm{t}_{\mathrm{i}} \approx 1\right)$. Os condutores mistos são aqueles onde parcelas significativas da condutividade são dadas por portadores iônicos e eletrônicos $\left(\mathrm{t}_{\mathrm{i}}+\mathrm{t}_{\mathrm{e}}=1\right)$.

Óxidos puros como $\mathrm{TiO}_{2}, \mathrm{SnO}_{2}, \mathrm{ZnO}, \mathrm{BaTiO}_{3}$ e $\mathrm{SrTiO}_{3}$, que podem ser facilmente reduzidos, são semicondutores do tipo n. Por outro lado, os óxidos que são facilmente oxidados, como os monóxidos dos metais de transição, apresentam condutividade eletrônica do tipo p. Entre os condutores mistos temos, por exemplo, o $\mathrm{MgO}$ que dependendo das condições de temperatura e de pressão parcial de oxigênio pode apresentar condutividade elétrica puramente eletrônica ou mista [1-3].

Um exemplo de condutor puramente iônico é o $\mathrm{ZrO}_{2}$ dopado com $\mathrm{Y}^{3+}$ ou $\mathrm{Ca}^{2+}$. A dopagem promove a substituição de íons $\mathrm{Zr}^{4+}$ pelo íon com menor valência, e para que o equilíbrio de cargas seja mantido são criadas vacâncias do íon oxigênio. Estas vacâncias são responsáveis pelo transporte elétrico nestes materiais, através de um processo termicamente ativado [1, 4].

O objetivo deste trabalho é descrever a montagem e calibração de um sistema de medidas elétricas que permite a realização de medidas elétricas em função da pressão parcial de oxigênio e da temperatura. A principal motivação de tais medidas é no desenvolvimento do método de Patterson (curvas $\left.\sigma=\mathrm{f}\left(\mathrm{p}_{\mathrm{O}_{2}}, \mathrm{~T}\right)\right)$. Nas cerâmicas condutoras iônicas este método permite a determinação de uma eventual contribuição de condução eletrônica e também a identificação do tipo de condução, tipo n ou p. Nas cerâmicas semicondutoras, ou naquelas em que as propriedades elétricas são determinadas pelos fenômenos intergranulares (varistores, termistores, por exemplo), este tipo de medida permite identificar o tipo de defeito majoritário nos contornos de grão responsável pelas propriedades elétricas do material.

\section{MONTAGEM EXPERIMENTAL}

A Fig. 1 apresenta um esquema da montagem experimental construída. O sistema consiste essencialmente de:

- um sensor de oxigênio, responsável pela determinação da pressão parcial de $\mathrm{O}_{2}\left(\mathrm{p}_{\mathrm{O}_{2}}\right)$ presente no gás vetor.

- uma bomba de oxigênio, responsável pelo enriquecimento ou empobrecimento em oxigênio do gás vetor (argônio ou nitrogênio).

- uma câmara de medidas estanque, impermeável aos gases, com um porta amostras para medidas seqüenciais em até três amostras.

Além dos componentes principais citados, também fazem parte do sistema construído os fornos do sensor, da bomba e da câmara de medidas, um milivoltímetro, uma fonte de corrente e as conexões e tubulações estanques em aço inox.

Este sistema, em conjunto com um analisador de impedância, permite a realização de medidas elétricas em função da temperatura e da pressão parcial de oxigênio. Com esta montagem, a temperatura pode ser variada entre a temperatura ambiente e $1000^{\circ} \mathrm{C}$ e a pressão parcial de oxigênio pode ser variada de forma controlada e continuamente entre $10^{-6}(1 \mathrm{ppm})$ e $1 \mathrm{~atm}$.

\section{Sensor eletroquímico de oxigênio}

Este dispositivo é composto de um tubo de zircônia estabilizada com ítria (YSZ), com uma de suas extremidades 


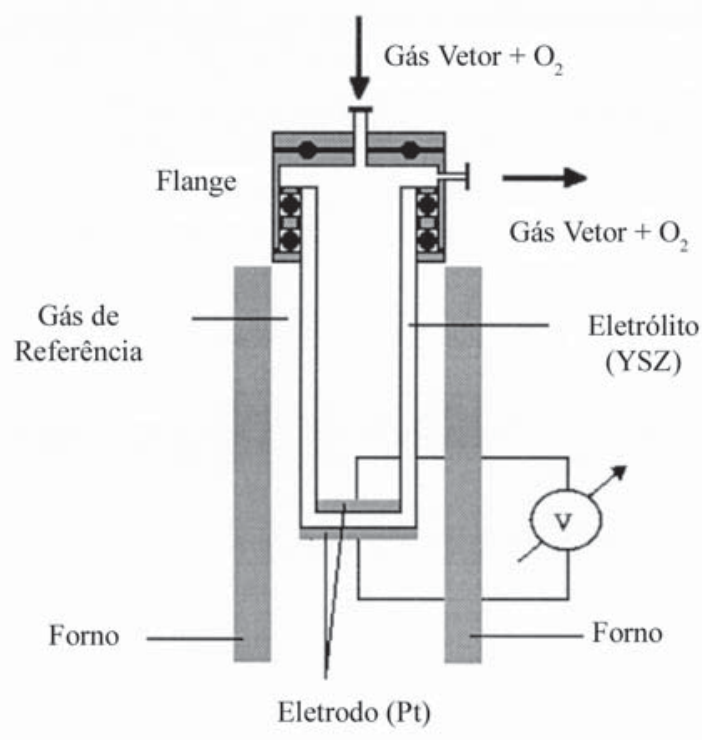

Figura 2: Diagrama esquemático do sensor de oxigênio. V: milivoltímetro.

[Figure 2: Schematics of the oxygen sensor. V: millivoltmeter.]

fechada (Fig. 2). Este tubo de zircônia é o eletrólito sólido do sensor. Na extremidade fechada do tubo foram depositados eletrodos de platina nas faces interna e externa. No interior do tubo circula o gás que será monitorado pelo sensor, enquanto que a face externa do tubo está em contato com o ar ambiente. Neste caso, o eletrodo interno será o eletrodo de trabalho (atmosfera de medida) e o eletrodo externo o eletrodo de referência (atmosfera conhecida). O sensor é inserido em um forno tubular com o intuito de garantir a temperatura adequada de operação (entre $800{ }^{\circ} \mathrm{C}$ e $900{ }^{\circ} \mathrm{C}$ ).

$\mathrm{O}$ princípio de funcionamento de um sensor eletroquímico de oxigênio é baseado na lei de Nernst. Quando um eletrólito sólido é colocado entre duas atmosferas, contendo diferentes teores de oxigênio, uma força eletromotriz (fem) é gerada entre as faces do eletrólito. A relação entre a fem e o gradiente de pressão de oxigênio é dada pela lei de Nernst:

$$
\mathrm{E}=\frac{\mathrm{RT}}{4 \mathrm{~F}} \ln \left(\frac{\mathrm{p}_{\mathrm{O}_{2}}}{\mathrm{p}_{\mathrm{ref}}}\right)
$$

onde E é a força eletromotriz do sensor, medida em Volt; $\mathrm{T}$ a temperatura absoluta do sensor; $\mathrm{p}_{\text {ref }}$ é a pressão parcial de oxigênio na atmosfera de referência (no caso do sensor construído, a atmosfera de referência é o ar exterior ao tubo, $\mathrm{p}_{\text {ref }}$ $=0,21 \mathrm{~atm}) ; \mathrm{p}_{\mathrm{O}_{2}}$ é a pressão parcial de oxigênio a ser determinada na atmosfera de medida; $\mathrm{R}$ é a constante dos gases perfeitos (8,3145 J/g.mol.K) e F é a constante de Faraday (96485,31 C/ g.mol). Portanto, através desta lei, conhecendo-se $\mathrm{T}$ e $\mathrm{p}_{\text {ref }}$ e medindo-se E, pode-se determinar a pressão parcial de oxigênio no gás vetor que circula no interior do tubo de zircônia.

\section{Bomba eletroquímica de oxigênio}

A bomba eletroquímica de oxigênio é um dispositivo que permite a introdução ou a extração de oxigênio em um gás ou uma atmosfera [5]. O dispositivo é constituído de um tubo de zircônia estabilizada com ítria, no qual foram depositadas camadas de platina nas superfícies interna e externa (Fig. 3). O gás vetor $\left(\mathrm{N}_{2}\right.$ ou Ar) circula no interior do tubo e o eletrodo exterior está em contato com o ar ambiente. O tubo se encontra no interior de um forno para garantir a temperatura de funcionamento da bomba (entre $700{ }^{\circ} \mathrm{C}$ e $900{ }^{\circ} \mathrm{C}$ ). Este dispositivo é empregado para controlar a pressão parcial de oxigênio no gás em circulação, ou seja, na realização de misturas gás vetor (inerte) com oxigênio.

Analogamente ao sensor de oxigênio, o funcionamento de uma bomba eletroquímica de oxigênio é baseado nas propriedades de condução iônica da zircônia estabilizada. A lei de Faraday relaciona a corrente elétrica I aplicada nas faces do eletrólito e o fluxo de oxigênio $\left(\mathrm{J}_{\mathrm{O}_{2}}\right)$ que atravessa o eletrólito:

$$
\mathrm{J}_{\mathrm{O}_{2}}=\frac{\mathrm{I}}{4 . \mathrm{F}}
$$

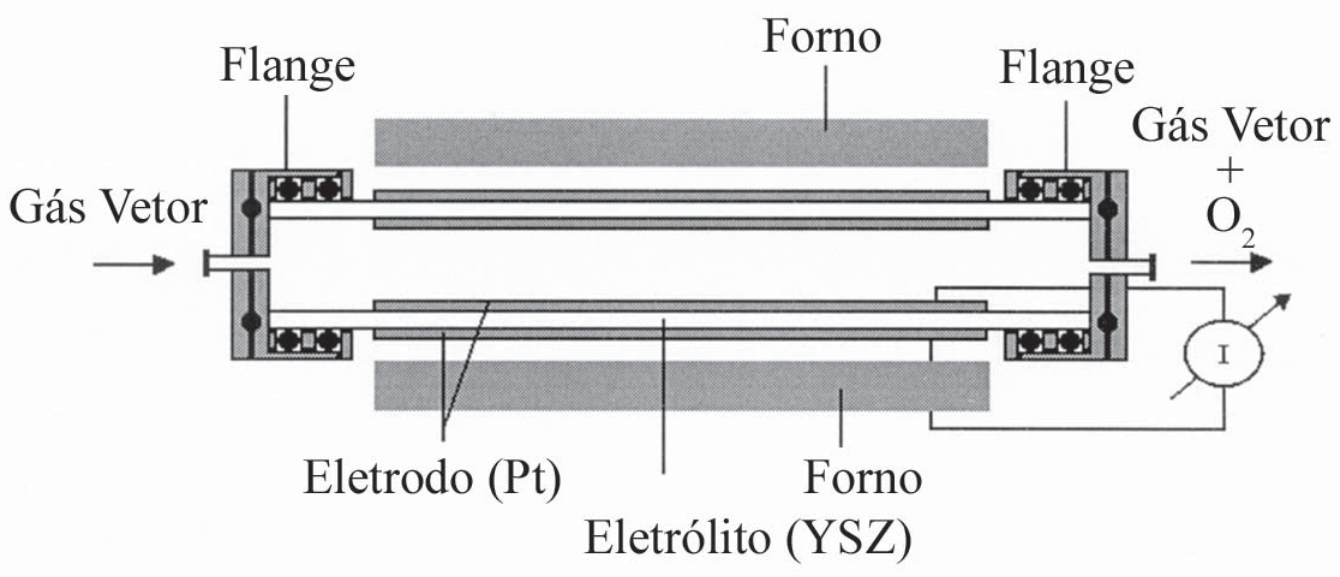

Figura 3: Diagrama esquemático da bomba eletroquimica de oxigênio. I: fonte de corrente para a bomba. [Figure 3: Schematics of the oxygen electrochemical pump. I: current supply.] 
Desta forma, o fluxo de $\mathrm{O}_{2}\left(\mathrm{~J}_{\mathrm{O}_{2}}\right)$ tem uma dependência linear com a intensidade da corrente aplicada. Para intensidades de corrente elétrica de até $\sim 1 \mathrm{~A}$, a equação B pode ser convertida para expressar diretamente a dependência da pressão parcial de oxigênio no gás vetor, circulando no interior da bomba, com a intensidade da corrente aplicada através da seguinte expressão [6]:

$$
\mathrm{p}_{\mathrm{O}_{2}}=\mathrm{C}+\left(\frac{0,209}{\mathrm{D}}\right) \mathrm{I}
$$

onde $\mathrm{p}_{\mathrm{O}_{2}}(\mathrm{~atm}), \mathrm{D}(\mathrm{L} / \mathrm{h}) \mathrm{e} \mathrm{I}(\mathrm{A})$, correspondem à pressão parcial de oxigênio, ao fluxo do gás vetor e a intensidade da corrente aplicada, respectivamente. A pressão parcial de oxigênio na mistura de gases resultante, saindo da bomba, varia linearmente com a intensidade de corrente. Portanto, utilizando-se esta relação é possível controlar continuamente e com boa precisão a quantidade do oxigênio que é injetada no gás vetor.

\section{Câmara de medidas}

A câmara de medidas é composta por um tubo de alumina (Degussa), estanque aos gases, e por duas flanges de aço inox colocadas nas extremidades do tubo. Estas flanges, refrigeradas a água, servem de suporte para o porta-amostras e possibilitam a circulação de gás no interior do tubo de alumina. A junção entre as peças metálicas e o tubo cerâmico é feita através de anéis elásticos, que garantem a estanqueidade da câmara de medidas.

A câmara de medidas é colocada no interior de um forno resistivo tubular vertical e as flanges metálicas permanecem fora da zona quente do forno. O porta-amostras (Fig. 4) é introduzido no interior do tubo de alumina através da flange superior. Esta câmara de medidas, usada em conjunto com um analisador de impedância e o forno, possibilita que sejam realizadas medidas elétricas em temperaturas entre a temperatura ambiente $\mathrm{e} \sim 1000{ }^{\circ} \mathrm{C}$.

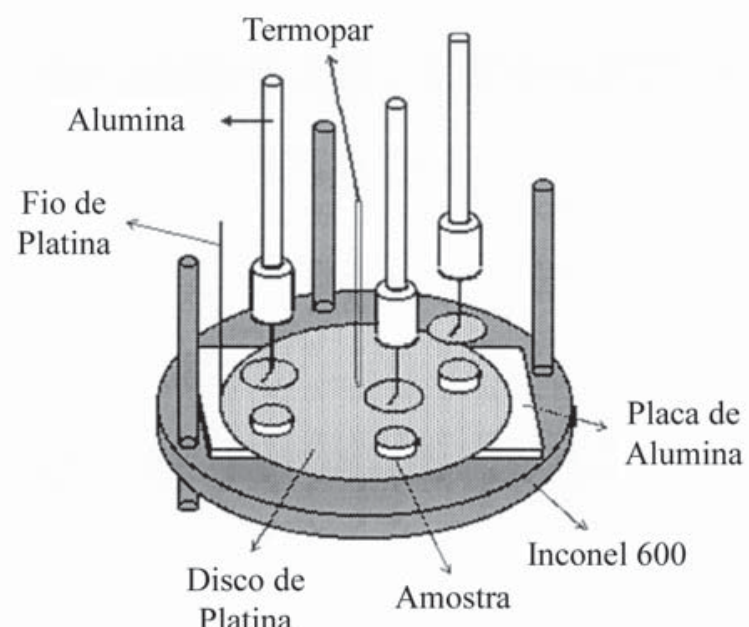

Figura 4: Diagrama esquemático do porta-amostra utilizado na câmara de medidas elétricas.

[Figure 4: Schematics of the sample holder of the sample chamber.]

\section{Calibração do Sistema de Medidas}

A calibração do sistema de controle da pressão parcial de oxigênio também é uma verificação direta do seu bom funcionamento e foi dividida em duas etapas. A primeira consiste em dois testes que permitem a verificação do funcionamento correto do sensor e da bomba de oxigênio. A segunda etapa é dedicada a verificação da estanqueidade da câmara de medidas.

\section{Lei de Nernst}

Teoricamente, quando os eletrodos do sensor estão em equilíbrio com o gás, a força eletromotriz $\mathrm{E}$ do sensor é dada pela lei de Nernst (equação A). Se as pressões $\mathrm{p}_{\mathrm{O}_{2}}$ e $\mathrm{p}_{\text {ref }}$ são mantidas constantes, então a tensão $\mathrm{E}$ é proporcional à temperatura absoluta T. O primeiro teste consiste em verificar esta proporcionalidade, ou seja, em estudar a variação da fem do sensor com a temperatura para uma determinada pressão parcial de oxigênio no gás a ser analisado.

$\mathrm{Na}$ verificação desta lei utilizou-se o nitrogênio como gás vetor. A Fig. 5 apresenta a força eletromotriz do sensor em função da temperatura de funcionamento do sensor medida entre $650{ }^{\circ} \mathrm{C}$ e $900{ }^{\circ} \mathrm{C}$. Para as temperaturas entre $700{ }^{\circ} \mathrm{C}$ e $880{ }^{\circ} \mathrm{C}$, a curva $\mathrm{E}(\mathrm{T})$ tem uma dependência linear com a temperatura, indicando o bom funcionamento do sensor nestas condições. Pode-se observar que para temperaturas menores que $\sim 700{ }^{\circ} \mathrm{C}$ e para temperaturas maiores que $\sim 880^{\circ} \mathrm{C}$ há um desvio da lei de Nernst. Em baixas temperaturas, este desvio é observado pois a impedância do sensor é elevada, devido à relativa baixa condutividade do eletrólito de zircônia estabilizada, e se aproxima da impedância de entrada do milivoltímetro usado para a medida. Portanto, uma corrente parasita pode surgir no sensor perturbando a medida da força eletromotriz. Em altas temperaturas, o desvio da linearidade está relacionado ao efeito da semi-permeabilidade eletroquímica da zircônia [7]. Este fenômeno é particularmente importante devido à baixa pressão parcial de $\mathrm{O}_{2}$ usada neste teste $\left(\sim 10^{-6} \mathrm{~atm}\right)$ em relação à pressão parcial da referência do sistema $\left(\mathrm{p}_{\text {ref }}=0,21 \mathrm{~atm}\right)$. A semipermeabilidade do eletrólito gera uma polarização do eletrodo de trabalho (ou de medida) que diminui o valor da fem medida em altas temperaturas.

Este teste da lei de Nernst é indicado pela IUPAC (International Union of Pure and Applied Chemistry) para se verificar o funcionamento correto de sondas eletroquímicas. A vantagem é não ser necessário o emprego de nenhum outro dispositivo além do sensor que é o objeto a ser aferido. Outro aspecto importante é que através dele pode-se determinar a faixa de temperatura de operação mais apropriada, que no caso presente é em torno de $800{ }^{\circ} \mathrm{C}$.

\section{Lei de Faraday}

Como descrito anteriormente, quando uma bomba eletroquímica funciona corretamente o fluxo de oxigênio que atravessa as paredes do eletrólito obedece a lei de Faraday (equação B) . Nas condições experimentais com fluxo de gás $\mathrm{D}$ constante, a equação $\mathrm{C}$ define uma relação linear entre $\mathrm{p}_{\mathrm{O}_{2}} \mathrm{e}$ 


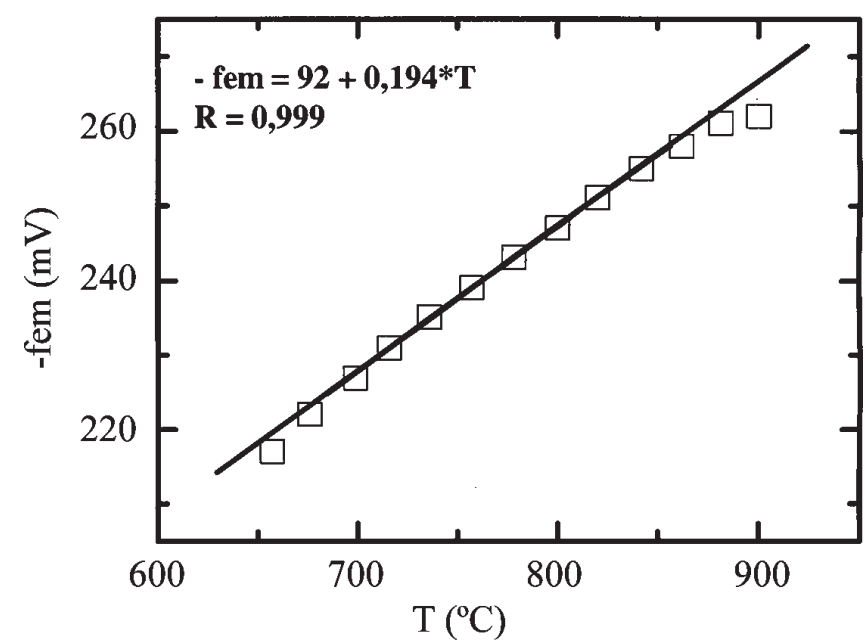

Figura 5: Dependência da força eletromotriz (fem) do sensor de oxigênio com a temperatura de funcionamento do sensor (lei de Nernst). A reta e os parâmetros ajustados correspondem ao ajuste linear dos valores experimentais.

[Figure 5: Oxygen sensor emf as as function of the sensor temperature - Nernst law.]

I. O segundo teste realizado consiste na verificação desta linearidade. Este teste também permite mostrar que o coeficiente angular da reta $\mathrm{P}_{\mathrm{O}_{2}} \mathrm{x}$ I corresponde à razão entre a $\mathrm{pO}_{2}$ no ar e o fluxo real $\mathrm{D}$ que sai do cilindro do gás vetor. Portanto, a partir do coeficiente angular $\alpha$ da reta ajustada podese calcular o fluxo de gás que atravessa a bomba e que circula em todo o sistema de medidas através da relação: $\mathrm{D}=0,209 / \alpha$. Este teste foi realizado com uma montagem utilizando-se o gás vetor nitrogênio, o sensor de oxigênio para medida da $\mathrm{p}_{\mathrm{O}_{2}}$, descrito anteriormente e uma fonte de corrente (TTi - PL320) para variação da corrente elétrica I que é injetada na bomba.

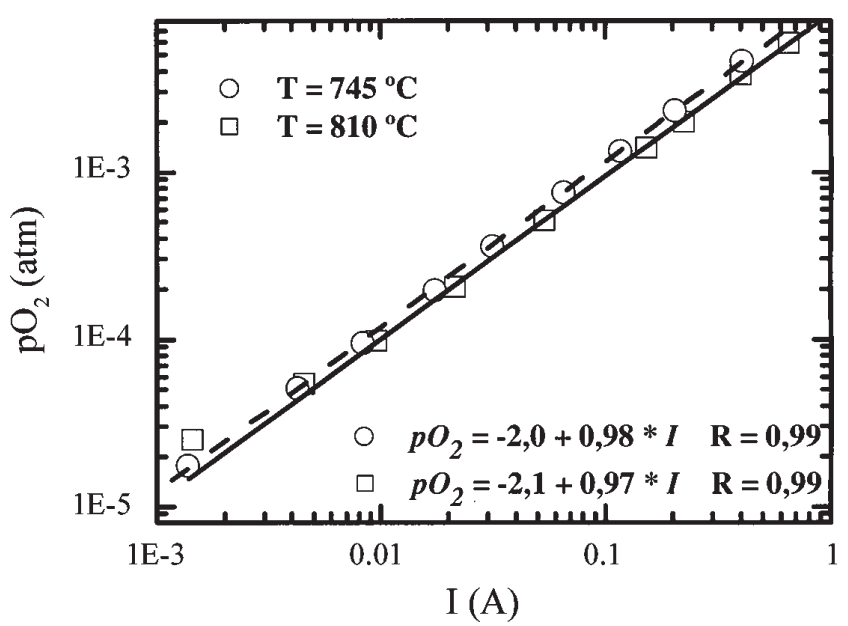

Figura 6: Dependência da pressão parcial de oxigênio injetada pela bomba eletroquímica no gás vetor com a corrente elétrica aplicada na bomba (lei de Faraday) medida a $745{ }^{\circ} \mathrm{C}$ e $810{ }^{\circ} \mathrm{C}$. As retas e os parâmetros ajustados correspondem aos ajustes lineares dos valores experimentais.

[Figure 6: Partial pressure of oxygen produced by the oxygen pump as a function of the electrical current supplied to the pump - Faraday's law, measured at $745^{\circ} \mathrm{C}$ and $810^{\circ} \mathrm{C}$.]
A Fig. 6 mostra a variação de $\mathrm{p}_{\mathrm{O}_{2}}$ com I para a bomba eletroquímica funcionando em duas temperaturas, 745 e $810^{\circ} \mathrm{C}$. O comportamento linear é observado em toda a faixa de corrente para as medidas realizadas a $745{ }^{\circ} \mathrm{C}$. Por outro lado, para a temperatura de funcionamento da bomba de $810^{\circ} \mathrm{C}$ ocorre um pequeno desvio da linearidade na região de baixas correntes. Este desvio pode estar relacionado com o fenômeno de semipermeabilidade eletroquímica dos eletrólitos sólidos à base de zircônia [7].

Estas medidas permitiram a confirmação do bom funcionamento da bomba numa ampla faixa de corrente elétrica e mostraram que é possível realizar misturas do tipo gás inerte (vetor) + oxigênio de forma controlada ente $10^{-6} \mathrm{e} 10^{-2}$ atm de $\mathrm{O}_{2}$.

\section{Estanqueidade da câmara de medidas}

Para se testar a estanqueidade da câmara de medidas o seguinte procedimento foi adotado: inicialmente, a câmara de medida foi retirada do circuito, e foram testados os demais componentes; o circuito constituído pelo sensor, bomba e todas as conexões foi submetido a um fluxo constante de Ar e a força eletromotriz no sensor foi medida. $\mathrm{O}$ valor encontrado indicou a presença de $\sim 1 \mathrm{ppm}\left(10^{-6} \mathrm{~atm}\right)$ de oxigênio, que corresponde ao limite inferior de deteç̧ão de oxigênio no sistema. Este teste indica que o conjunto bomba-sensor e conexões não apresenta uma contaminação com oxigênio detectável ( $\leq 1 \mathrm{ppm})$. Um segundo teste realizado consistiu em introduzir a câmara de medida no sistema e sob um fluxo de argônio, medir a força eletromotriz no sensor. Neste caso, o valor medido indicou a presença de $\sim 10$ ppm de oxigênio.

\section{Caracterização de amostras}

Como exemplo da utilização do sistema de medidas elétricas com atmosfera controlada, resultados preliminares de dois trabalhos de pesquisa em andamento são apresentados.

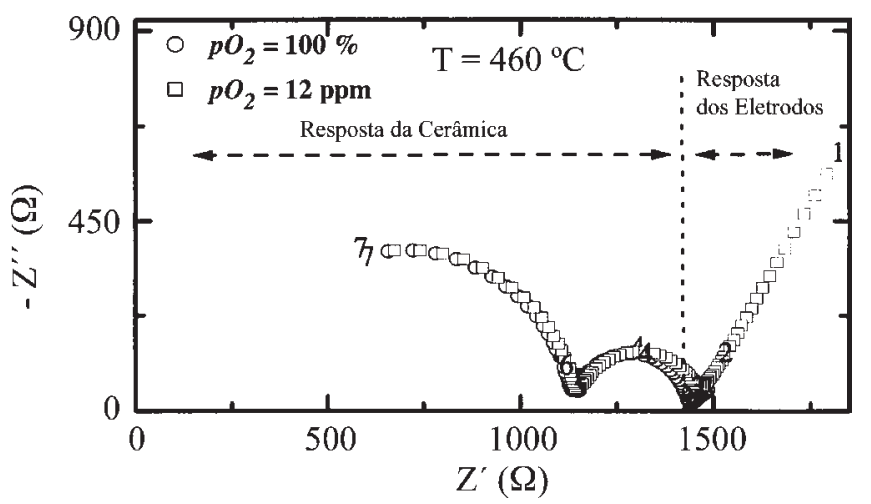

Figura 7: Diagramas de impedância de zircônia estabilizada (9 mol\% de $\mathrm{Y}_{2} \mathrm{O}_{3}$ ) obtidos à $460{ }^{\circ} \mathrm{C}$ em duas atmosferas de oxigênio: $\mathrm{p}_{\mathrm{O}_{2}}=$ $100 \%$ (○) e 12 ppm ( $\square$ ). Nos diagramas de impedância, os números indicam o logaritmo da frequência do sinal elétrico.

[Figure 7: Impedance diagrams of 9 mol\% yttria-stabilized zirconia measured at $460{ }^{\circ} \mathrm{C}$ under $100 \% \mathrm{O}_{2}(\mathrm{O})$ and $12 \mathrm{ppm} \mathrm{O}_{2}(\square)$. Numbers in the diagrams stand for the logarithm of frequency.] 
O primeiro exemplo é a caracterização de eletrólitos sólidos à base de zircônia. A Fig. 7 mostra os diagramas de impedância de uma amostra de YSZ (zircônia estabilizada com 9 mol\% de $\mathrm{Y}_{2} \mathrm{O}_{3}$ ) obtidos a $460{ }^{\circ} \mathrm{C}$ em duas atmosferas de oxigênio: $\mathrm{p}_{\mathrm{O}_{2}}=100 \%$ e $12 \mathrm{ppm}$. Pode-se observar que a parte do diagrama de impedância correspondente a resposta da cerâmica (região de altas frequências) não sofre alterações significativas. Esta observação está de acordo com as propriedades de condutor puramente iônico $\left(\mathrm{t}_{\mathrm{i}} \sim 1\right)$ deste material. Nestas condições de temperatura e pressão parcial de oxigênio, a condutividade elétrica da YSZ depende somente da concentração de vacâncias de oxigênio. Esta concentração de portadores iônicos é fixada pela concentração de dopante adicionada durante a elaboração do material e permanece constante durante a realização de medidas elétricas em função da $\mathrm{p}_{\mathrm{O}_{2}}$. Por outro lado, uma modificação na região de baixas frequências é observada. Esta região de frequências corresponde à resposta dos eletrodos (interface metal - cerâmica). A resposta dos eletrodos está associada à cinética da reação eletrodooxigênio $\left(1 / 2 \mathrm{O}_{2} \leftrightarrow \mathrm{O}^{2-}+2 \mathrm{e}^{-}\right)$que depende da quantidade de gás $\mathrm{O}_{2}$ presente nas interfaces.

$\mathrm{O}$ segundo material estudado foi um varistor à base de óxido de estanho. Este estudo das propriedades elétricas desta cerâmica foi realizado variando-se a temperatura e atmosfera de tratamentos térmicos. Inicialmente, as amostras foram tratadas a $850{ }^{\circ} \mathrm{C} / 1 \mathrm{~h}$ em $\mathrm{O}_{2}$ puro. Em seguida, foram realizadas medidas de espectroscopia de impedância nas temperaturas de $210{ }^{\circ} \mathrm{C} \mathrm{e} 460{ }^{\circ} \mathrm{C} \mathrm{em} \mathrm{O}$ puro. Após a medida a $460{ }^{\circ} \mathrm{C} \mathrm{em} \mathrm{O}_{2}$ puro, a atmosfera foi alterada para um fluxo de argônio ( 12 ppm de $\left.\mathrm{O}_{2}\right)$ e, depois da estabilização da atmosfera $(\sim 10 \mathrm{~h})$, foram realizadas novas medidas de espectroscopia de impedância a $460{ }^{\circ} \mathrm{C}$ e $210{ }^{\circ} \mathrm{C}$ nesta atmosfera. A Fig. 8 mostra as medidas de espectroscopia de impedância a $460{ }^{\circ} \mathrm{C}$ em duas atmosferas de $\mathrm{O}_{2}(100 \%$ e 12 ppm). Estas medidas mostram que a resistência elétrica total da cerâmica diminui com a diminuição de $\mathrm{p}_{\mathrm{O}_{2}}$. Esta diminuição da condutividade elétrica está de acordo com as

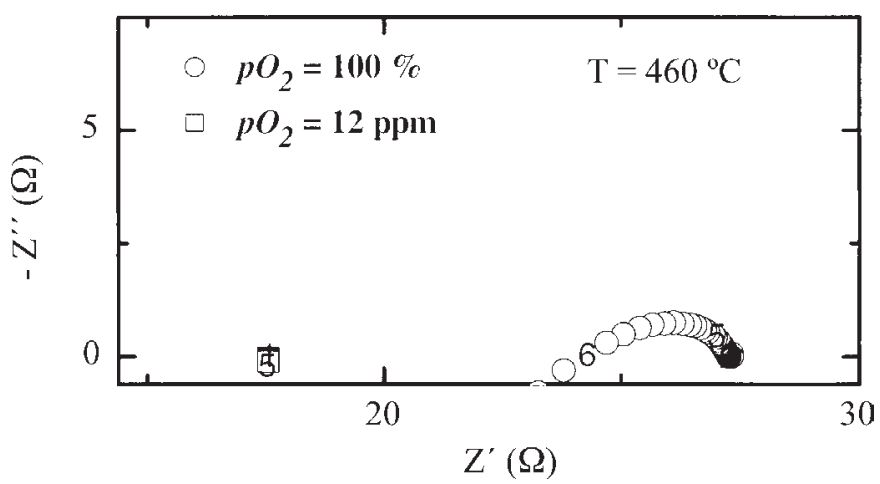

Figura 8: Diagramas de impedância de varistor à base de óxido de estanho medidos a $460{ }^{\circ} \mathrm{C}$ em duas pressões parciais de oxigênio $\mathrm{p}_{\mathrm{O}_{2}}=100 \%(\bigcirc)$ e $12 \mathrm{ppm}(\square)$.

[Figure 8: Impedance diagrams of a $\mathrm{SnO}_{2}$-based varistor measured at $460{ }^{\circ} \mathrm{C}$ under $100 \% \mathrm{O}_{2}(\mathrm{O})$ and $12 \mathrm{ppm} \mathrm{O}_{2}(\square)$. Numbers in the diagrams stand for the logarithm of frequency.]

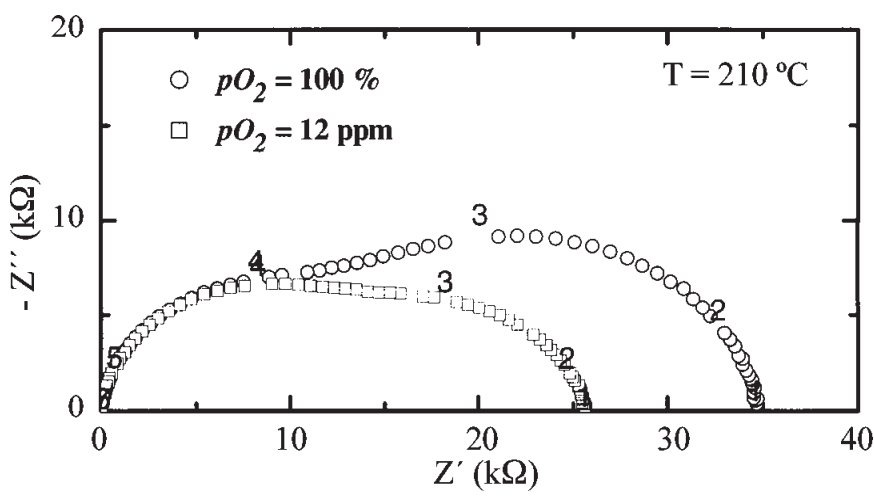

Figura 9: Diagramas de impedância de varistor à base de óxido de estanho medidos a $210{ }^{\circ} \mathrm{C}$ em duas pressões parciais de oxigênio $\mathrm{p}_{\mathrm{O}_{2}}=100 \%(\bigcirc)$ e $12 \mathrm{ppm}(\square)$.

[Figure 9: Impedance diagrams of a $\mathrm{SnO}_{2}$-based varistor measured at $210{ }^{\circ} \mathrm{C}$ under $100 \% \mathrm{O}_{2}(\mathrm{O})$ and $12 \mathrm{ppm} \mathrm{O}_{2}(\square)$. Numbers in the diagrams stand for the logarithm of frequency.]

propriedades de um semicondutor do tipo n, já verificada para o óxido de estanho [1]. Na Fig. 9 são mostrados os diagramas de impedância medidos a $210{ }^{\circ} \mathrm{C}$ nas duas atmosferas de $\mathrm{O}_{2}(100 \%$ e $12 \mathrm{ppm})$, nesta temperatura a faixa de freqüências permite a resolução de componentes do transporte elétrico. Pode-se observar que os diagramas de impedância (Fig. 9) são compostos de duas relaxações: uma em altas freqüências (AF), aproximadamente entre $10^{6}$ e $10^{4}$ $\mathrm{Hz}$, e outra em baixas freqüências (BF), aproximadamente entre 10 e $10^{4} \mathrm{~Hz}$. Os resultados mostram que a diminuição da resistência elétrica da amostra está relacionada, principalmente, com modificações na região de $\mathrm{BF}$ dos diagramas de impedância. Esta região de freqüências é normalmente relacionada com fenômenos intergranulares, indicando que os tratamentos térmicos realizados em diferentes atmosferas de oxigênio influenciam nas propriedades dos contornos de grão destes materiais [9]. Os resultados preliminares sugerem que estas medidas elétricas com controle da pressão parcial de oxigênio podem fornecer informações importantes sobre os mecanismos de condução elétrica nestes materiais.

\section{SUMÁRIO}

Este trabalho apresenta a montagem e calibração de um sistema de controle de pressão parcial de oxigênio. Este sistema, utilizado em conjunto com um analisador de impedância, permite o estudo das propriedades elétricas de diferentes materiais em amplas faixas de temperatura, pressão parcial de oxigênio, freqüência e amplitude do sinal elétrico aplicado. Os dispositivos eletroquímicos construídos (sensor e bomba) podem gerar e monitorar pressões parciais de oxigênio entre $10^{-6}$ e 1 atm de forma contínua e com boa precisão. Os testes realizados com o sensor e a bomba permitiram verificar experimentalmente as Leis de Nernst e de Faraday, respectivamente, indicando o bom funcionamento dos componentes desta montagem. Os resultados apresentados de caracterização elétrica de cerâmicas evidenciaram que a montagem experimental construída pode fornecer informações importantes nos 
estudos das propriedades elétricas destes materiais.

\section{AGRADECIMENTOS}

À FAPESP (Projeto Temático 99/10798-0) pelo financiamento. M. C. Steil agradece à FAPESP pela bolsa de pesquisador visitante (Proc. 02/03201-2). J. F. Q. R. e F. C. F. são bolsistas FAPESP Procs. 01/14033-0 e 01/04231-0, respectivamente. A M. Henault do LEPMI, Grenoble, França, pelo suporte técnico. Ao LIEC-CMDMC-UFSCar pela cessão de amostras de varistores. Este trabalho faz parte do Acordo de Cooperação de Pesquisa entre a École Nationale Supérieure de Chimie de Lille, França e o IPEN (2000-2004).

\section{REFERÊNCIAS}

[1] Y-M. Chiang, Dunbar Birnie III e W. D. Kingery, Physical Ceramics - Principles for Ceramic Science Engineering, The
MIT series in Materials Science and Engineering, John Wiley and Sons, Inc. New York (1997) Cap.3.

[2] D. Semposlinsk, W. D. Kingery, J. Am. Ceram. Soc. 63 (1980) 664.

[3] D. Semposlinsk, W. D. Kingery, H. L. Tuller, J. Am. Ceram. Soc. 63 (1980) 669.

[4] I. W. Chen, P. Li, J. Penner-Hanh, Mat. Res. Soc. Symp. Proc. 307 (1993) 27.

[5] J. Fouletier, G. Vitter, M. Kleitz, J. Appl. Electrochem. 5 (1975) 111.

[6] Electrochimie de Solides, C. Deportes, M. Duclot, P. Fabry, J. Fouletier, A. Hammou, M. Kleitz, E. Siebert e J. Souquet, Presses Universitaires de Grenoble, Grenoble (1994).

[7] J. Fouletier, P. Fabry, M. Kleitz, J. Electrochem. Soc. 123 (1976) 204.

[8] W. K. Bacelar, M. M. Oliveira, V. C. Souza, E. Longo, E. R. Leite, J. A. Varela, J. Mater. Sci. - Materials in Electronics 13 (2002) 409.

(Rec. 05/09/02, Ac. 09/09/02) 